\title{
DEVELOPMENT OF A TRANSPORTABLE LASER COOLED STRONTIUM SOURCE FOR FUTURE APPLICATIONS IN SPACE
}

\author{
M. Schioppo, G.M. Tino, N. Poli, M.G. Tarallo, D.V. Sutyrin \\ Dipartimento di Fisica and LENS, Università di Firenze, INFN Sezione di Firenze, \\ via Sansone 1, 50019 Sesto Fiorentino, Italy \\ guglielmo.tino@fi.infn.it
}

Ch. Lisdat, J.S.R. Vellore Winfred, S. Falke, U. Sterr, T. Legero, F. Riehle, Physikalisch-Technische Bundesanstalt, Bundesallee 100, 38116 Braunschweig, Germany

uwe.sterr@ptb.de

L. Cacciapuoti

ESA-ESTEC Noordwijk, The Netherlands

Luigi.Cacciapuoti@esa.int

\section{INTRODUCTION}

Optical clocks have recently reached levels of performance that are an order of magnitude or more beyond those of their microwave counterparts, which have historically set the standard for precision time/frequency metrology [1, 2. With this level of precision scientists are performing new and more stringent tests of fundamental physical principles, such as searches for temporal drifts in the fundamental constants [3, 4. But Earth-based operation of near-future optical clocks (with $10^{-18}$ fractional instability and accuracy level) will be limited by uncertainties in the value of the gravitational potential at the clocks locations and in their relative velocity caused, e.g. by continental drift [5]. For applications requiring the highest time and frequency precision it is therefore essential to operate optical clocks in Space, where the above effects can be determined with sufficient accuracy, by continuously measuring the orbit parameters. Furthermore, the operation of optical clocks in Space provides new scientific and technological opportunities [6]. In particular, an Earth-orbiting satellite containing an ensemble of optical clocks would allow a precision measurement of the gravitational redshift, navigation with improved precision, mapping of the Earth's gravitational potential by relativistic geodesy, and comparisons between ground clocks [7. In this proceeding we show the first step toward a Space optical clock by developing a transportable laser cooled strontium source that it will be integrated in a fully transportable optical lattice

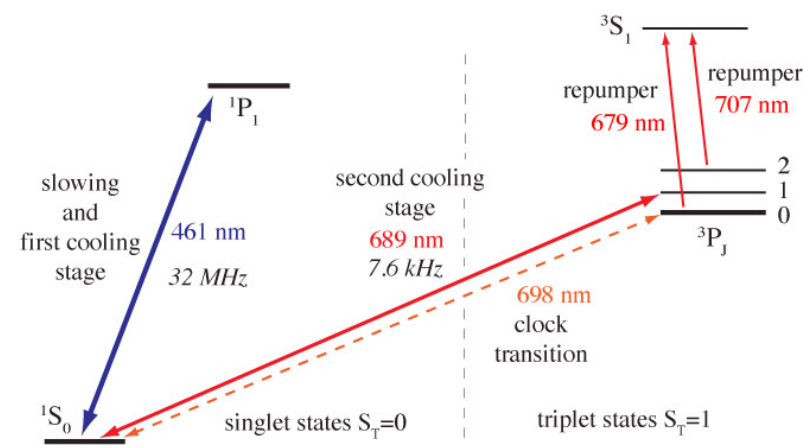

Figure 1: Partial level scheme of strontium. Only the transitions of interest are shown in the picture. The lattice magic wavelength for the clock ${ }^{1} S_{0}-{ }^{3} P_{0}$ transition strontium atoms is $813 \mathrm{~nm}$. 
strontium clock. This work is done in the framework of the ESA funded "SOC" Space Optical Clocks project, with the final aim to provide specifications for a future engineered model for applications in Space. Such a transportable optical clock can be used on Earth to characterize other optical frequency standards placed in stationary laboratories around the world, toward the future re-definition of the SI second based on optical standards. For all these purposes a compact, robust, and versatile version of optical atomic clock is necessary. In this sense the Sr lattice optical clock seems to be the most promising, for two main reasons: 1) all the needed lights for the clock operation (cooling, repumping, clock and lattice laser) can be produced by semiconductor lasers [8, 9, 10, 11, 12, (see Fig 1), 2) the Sr lattice clock is the most mature in therms of technology and scientific results with many under development around the world. In a recent demonstration, a Sr lattice clock achieved a fractional short term instability of $10^{-15}$ for an averaging time of $1 \mathrm{~s}$ [13, 14, 15] and an absolute fractional frequency uncertainty of $10^{-17}[16$.

\section{MAIN REQUIREMENTS AND DESIGN SOLUTIONS}

The main components to produce a ${ }^{88} \mathrm{Sr}$ sample of about $10^{8}$ atoms at $1 \mathrm{mK}$ temperature are: cooling laser at $461 \mathrm{~nm}$ (optical power $\gtrsim 200 \mathrm{~mW}$ ), high flux collimated atomic beam, vacuum system (pressure $10^{-8} \div 10^{-9}$ torr), proper designed magnetic fields for slowing the atomic beam and trapping the atoms in a magneto-optical trap, opto-mechanics (mirrors, cubes, plates and AOMs). For a transportable system all these elements must be assembled taking into account the requests of:

- compact design

- hardware modularity

- low power consumption

- operation reliability

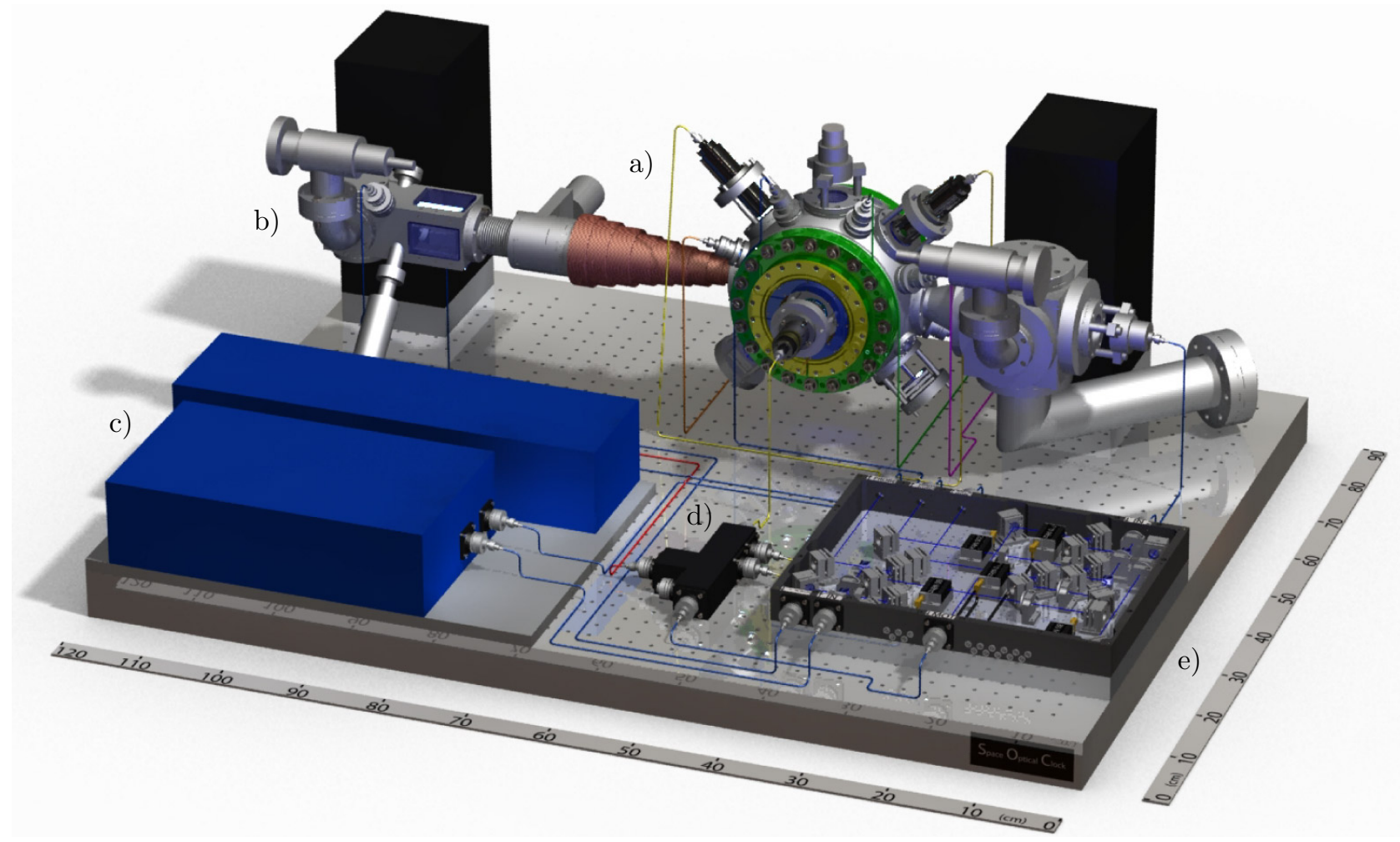

Figure 2: 3D model of the transportable cold strontium source. a) Vacuum system. b) High efficiency oven. c) Blue laser at $461 \mathrm{~nm}$. d) Dichroic fiber port cluster. e) Compact breadboard for the opto-mechanics. 
To match all these requirements several design solutions have been adopted:

- the vacuum system has been engineered according to the magnetic fields needed to slow and trapping the atoms

- new high-efficiency and low power consumption atomic oven has been designed

- all the opto-mechanics has been confined in a compact breadboard

- all the needed lights are delivered to the vacuum system by single mode optical fibers

- a dichroic fiber port cluster is used to couple into the same fibers both the lights needed for the first and the second cooling stage (including stirring beams for fermionic isotope).

The resulting 3D model in Fig 2 shows that the cold strontium source can be mounted on a main breadboard $1.2 \mathrm{~m} \times 0.9 \mathrm{~m}$, filling a volume of about 210 liters (without electronics).

\section{CURRENT STATUS AND EXPERIMENTAL RESULTS}
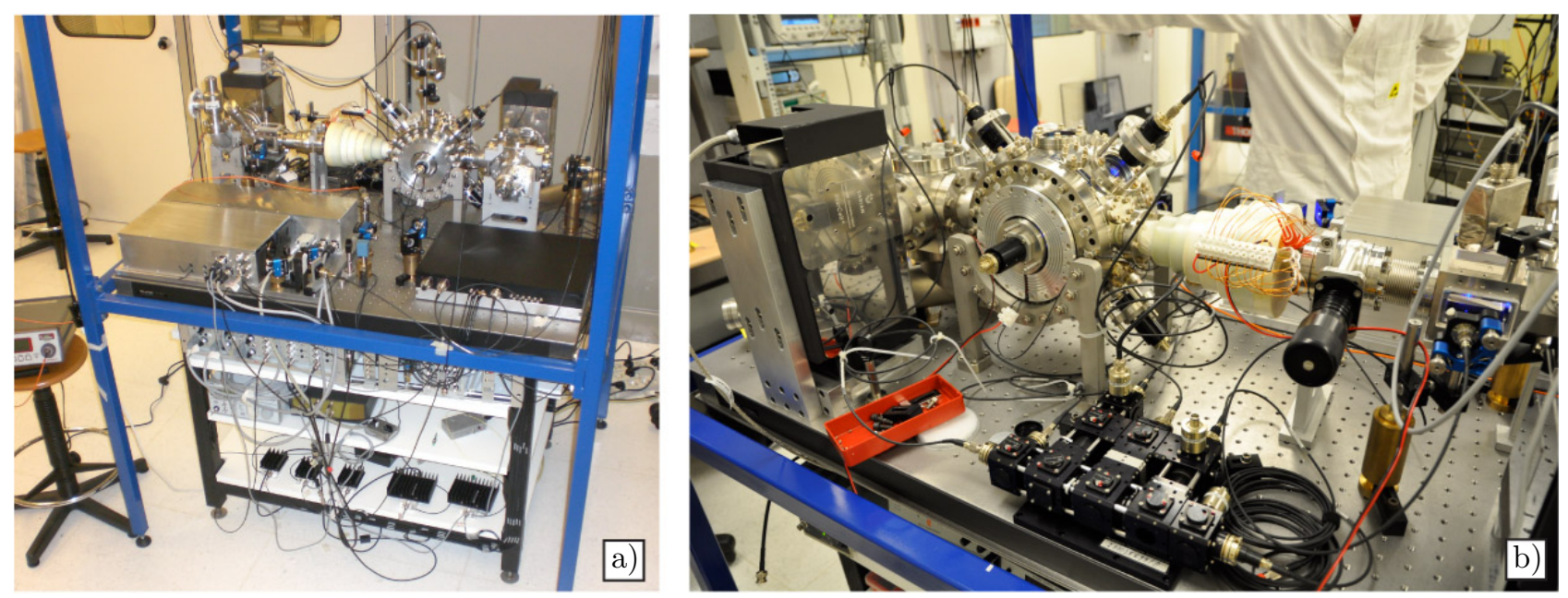

Figure 3: Current status of the transportable cold strontium source from different points of view. a) Total view of the setup showing the "atomic-package" (vacuum system, blue laser, compact breadboard, dichroic fiber cluster) and the consoles hosting all the electronics for the real-time experiment control. b) Rear view of the atomic-package showing the detail of the dichroic fiber port cluster.

After three years of work most of the components of the transportable cold strontium source have been completed. Fig 3 shows the current status of the system from different points of view. All the electronics for the control of the experiment is hosted under the main breadboard in order to provide a defined total volume of about 1200 liters (see Tab1). This value is given by the sum of the volume filled by: electronics (not yet optimized occupying about 920 liters), main breadboard (70 liters, that here works only as fastening base) and "atomic-package" (given by vacuum system, blue laser, compact breadboard and fiber port cluster) with an occupation of only 210 liters. This volume represents a factor of reduction of about 10 with respect to a standard apparatus. Mass and power consumption have been reduced of about a factor 3 and 5 respectively.

The system is currently under characterization. The first part of the characterization has been focused on the atomic oven. The working temperature of about $370{ }^{\circ} \mathrm{C}$ have been reached with only $15 \mathrm{~W}$ of power consumption. The atomic beam produced at this temperature has been characterized by performing a transverse spectroscopy with an orthogonal laser beam at $461 \mathrm{~nm}$. By detecting the fluorescence signal an atomic flux of about $3 \cdot 10^{11}$ atoms/sec has been estimated. 


\begin{tabular}{|l|c|c|c|}
\hline Transportable system & Volume $(\mathbf{l})$ & Mass $(\mathrm{kg})$ & Power (W) \\
\hline 1. vacuum system & $120 \mathrm{~cm} \times 40 \mathrm{~cm} \times 36 \mathrm{~cm} \simeq 170$ & 80 & 80 \\
2. blue laser & $38 \mathrm{~cm} \times 57 \mathrm{~cm} 12 \mathrm{~cm} \simeq 25$ & 20 & 25 \\
3. compact breadboard & $30 \mathrm{~cm} \times 40 \mathrm{~cm} \times 7.6 \mathrm{~cm}=9.1$ & 15 & 5 \\
4. dichroic cluster & $30 \mathrm{~cm} \times 20 \mathrm{~cm} \times 10 \mathrm{~cm}=6$ & 5 & 0 \\
5. main breadboard & $120 \mathrm{~cm} \times 90 \mathrm{~cm} \times 6.5 \mathrm{~cm}=70$ & 100 & 0 \\
6. electronics & $120 \mathrm{~cm} \times 90 \mathrm{~cm} \times 85 \mathrm{~cm} \simeq 920$ & 20 & 20 \\
total & $\mathbf{1 2 0 0}$ & $\mathbf{2 4 0}$ & $\mathbf{1 3 0}$ \\
atomic-package $(1 .+2 .+3 .+4)$. & $\mathbf{2 1 0}$ & $\mathbf{1 2 0}$ & $\mathbf{1 1 0}$ \\
\hline
\end{tabular}

\begin{tabular}{|l|c|c|c|}
\hline Stationary system & Volume (l) & Mass (kg) & Power (W) \\
\hline total & $200 \mathrm{~cm} \times 200 \mathrm{~cm} \times 150 \mathrm{~cm}=\mathbf{6 0 0 0}$ & $\mathbf{7 0 0}$ & $\mathbf{5 2 0}$ \\
atomic-package & $200 \mathrm{~cm} \times 200 \mathrm{~cm} \times 60 \mathrm{~cm}=\mathbf{2 4 0 0}$ & $\mathbf{3 0 0}$ & $\mathbf{5 0 0}$ \\
\hline
\end{tabular}

\begin{tabular}{|l|c|c|c|}
\hline compaction factor & Volume $(\mathrm{l})$ & Mass $(\mathrm{kg})$ & Power $(\mathbf{W})$ \\
\hline total & $6000 / 1200=\mathbf{5}$ & $700 / 240 \simeq \mathbf{3}$ & $520 / 130=\mathbf{4}$ \\
atomic-package & $2400 / 210 \simeq \mathbf{1 0}$ & $300 / 120=\mathbf{2 . 5}$ & $500 / 110 \simeq \mathbf{5}$ \\
\hline
\end{tabular}

Table 1: Final budget for volume, mass and power consumption achieved by the transportable setup in comparison with the analogous values obtained by a standard stationary system.
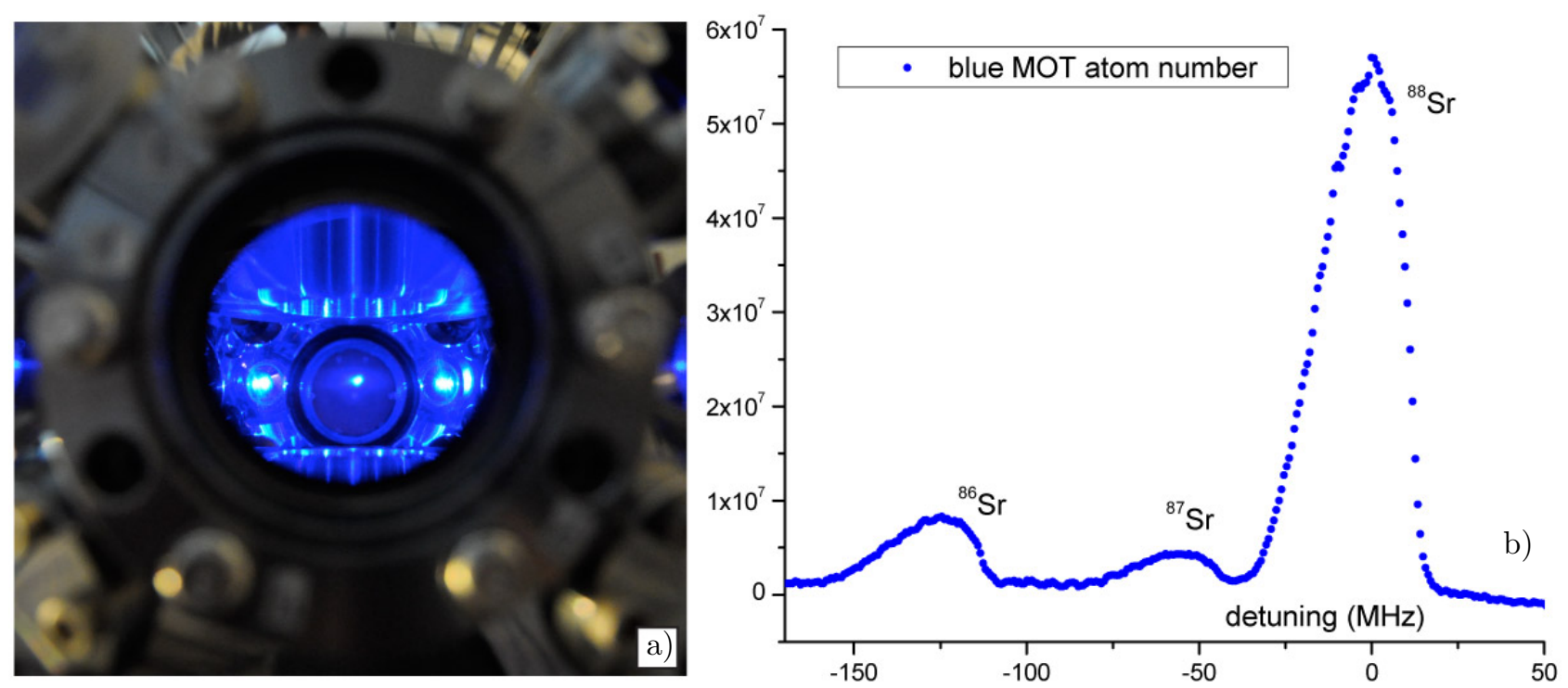

Figure 4: First magneto-optical trap (MOT) at $461 \mathrm{~nm}$ realized on the transportable setup. a) Picture of the ${ }^{88} \mathrm{Sr}$ blue MOT seen by one of the CF40 windows used to collect the atom's fluorescence. b) Plot of the fluorescence signal in function of the blue laser detuning. Together with the signal due to the most abundant ${ }^{88} \mathrm{Sr}(82.58 \%)$ also signals of ${ }^{87} \mathrm{Sr}(7.00 \%)$ and ${ }^{86} \mathrm{Sr}(9.86 \%)$ trapped atoms can be resolved.

The fluorescence light coming from atoms trapped in the magneto-optical trap (MOT) at $461 \mathrm{~nm}$ has been quite easily observed thanks to the high value of atomic flux coming from the oven. By detecting these fluorescence photons a number of trapped atoms of about $6 \cdot 10^{7}$ (see plot of Fig 4 ), at the Doppler temperature of about $1 \mathrm{mK}$, has been estimated. This result confirm the proper design of the magnetic fields for Zeeman slowing and MOT. Also laser beams intensity, geometry and alignment for the first cooling stage has been successfully tested. 


\section{CONCLUSION}

In this work we presented the first prototype of transportable laser-cooled strontium source. Novel design solutions have been discussed with a final volume, mass and power consumption budget for the atomic-package of 210 liters, $120 \mathrm{~kg}$ and $110 \mathrm{~W}$ respectively. Most of the home made components have been assembled and tested. The system is currently under characterization and it is already able to produce a sample of about $10^{8}$ atoms at $1 \mathrm{mK}$ temperature. Next steps will be the integration of this system with a second stage cooling laser and with a transportable clock laser in order to realize a fully transportable optical lattice clock. Technical solutions, methods and know-how developed in this work will be used to built more robust, compact and advanced technology apparatus, with the final aim to provide specifications for a future space optical lattice clock.

\section{ACKNOWLEDGMENTS}

This work was supported by ESA ("SOC" Space Optical Clocks project) and LENS. We thank F. Sorrentino, A. Giorgini and G. Rosi, working on "SAI" Space Atom Interferometer project, for useful discussions and suggestions.

\section{References}

[1] S. A. Diddams, J. C. Bergquist, S. R. Jefferts, C. W. Oates, "Standards of Time and Frequency at the Outset of the 21st Century", Science 306, 1318 (2004)

[2] M. Takamoto, F. L. Hong, R. Higashi and H. Katori, "An optical lattice clock", Nature 435, 321 (2005).

[3] S. Bize, S. A. Diddams, U. Tanaka, C. E. Tanner, W. H. Oskay, R. E. Drullinger, T. E. Parker, T. P. Heavner, S. R. Jefferts, L. Hollberg, W. M. Itano, J. C. Bergquist, "Testing the Stability of Fundamental Constants with the $199 \mathrm{Hg}^{+}$Single-Ion Optical Clock", Phys. Rev. Lett. 90, 150802 (2003)

[4] T. Rosenband, D.B. Hume, P.O. Schmidt, C.w. Chou, A. Brusch, L. Lorini, W.H. Oskay, R.E. Drullinger, T.M. Fortier, J.E. Stalnaker, S.A. Diddams, W.e. Swann, N.R. Newbury, W.M. ltano, D.J. Wineland, and J.C. Bergquist, "Frequency Ratio of $\mathrm{Al}^{+}$and $\mathrm{Hg}^{+}$Single-Ion Optical Clocks; Metrology at the 17th Decimal Place", Science 319, 1808 (2008)

[5] D. Kleppner, "Time Too Good to Be True", Physics Today 59, $10-11$ (2006). http://dx.doi.org/10.1063/1.2195297

[6] S. Schiller, A. Görlitz, A. Nevsky, J. C. J. Koelemeij, A. Wicht, P. Gill, H. A Klein, H. S. Margolis, G. Mileti, U. Sterr, F. Riehle, E. Peik, Chr. Tamm, W. Ertmer, E. Rasel, V. Klein, C. Salomon, G. M. Tino, P. Lemonde, R. Holzwarth, T. W. Hänsch, "Optical Clocks in Space”, Nuclear Physics B-Proceedings Supplements. 166, 300-302 (2007).

[7] S. Schiller, G. M. Tino, P. Gill, C. Salomon, U. Sterr, E. Peik, A. Nevsky, A. Görlitz, D. Svehla, G. Ferrari, N. Poli, L. Lusanna, H. Klein, H. Margolis, P. Lemonde, P. Laurent, G. Santarelli, A. Clairon, W. Ertmer, E. Rasel, J. Müller, L. Iorio, C. Lämmerzahl, H. Dittus, E. Gill, M. Rothacher, F. Flechner, U. Schreiber, V. Flambaum, Wei-Tou Ni, Liang Liu, Xuzong Chen, Jingbiao Chen, Kelin Gao, L. Cacciapuoti, R. Holzwarth, M. P. He, W. Schäfer, "Einstein Gravity Explorer-a medium-class fundamental physics mission", Experimental Astronomy 23, 573-610 (2009)

[8] G. Ferrari, P. Cancio, R. E. Drullinger, G. Giusfredi, N. Poli, M. Prevedelli, C. Toninelli and G. M. Tino, "Precision Frequency Measurement of Visible Intercombination Lines of Strontium", Phys. Rev. Lett. 91, $243002(2003)$

[9] N. Poli, G. Ferrari, M. Prevedelli, F. Sorrentino, R. E. Drullinger, G. M. Tino, "Laser sources for precision spectroscopy on atomic strontium", Spectrochim. Acta Part A 63, 981 (2006)

[10] N. Poli, R. E. Drullinger, G. Ferrari, M. Prevedelli, F. Sorrentino, M. G. Tarallo, and G. M. Tino, "Prospect for a compact strontium optical lattice clock" Proc. SPIE 6673, 66730F (2007). 
[11] T. Legero, J.S.R. Winfred, F. Riehle, U. Sterr, "Ultracold ${ }^{88} \mathrm{Sr}$ atoms for an optical lattice clock", IEEE Proc. EFTFFCS 119 (2007). doi:10.1109/FREQ.2007.4319045

[12] N. Poli, M. G. Tarallo, M. Schioppo, C. W. Oates, G. M. Tino, "A simplified optical lattice clock", Appl. Phys. B 97, 27-33 (2009).

[13] M. Takamoto, F.-L. Hong, R. Higashi, Y. Fuji, M. Imae, and H. Katori, "Improved frequency measurement of a one-dimensional optical lattice clock with a spin-polarized fermionic ${ }^{87}$ Sr isotope", J. Phys. Soc. Jap. 75, pp. 104302-1-10 (2006).

[14] X. Baillard, M. Fouché, R. Le Targat, P. G. Westergaard, A. Lecallier, F. Chapelet, M. Abgrall, G. D. Rovera, P. Laurent, P. Rosenbusch, S. Bize, G. Santarelli, A. Clairon, P. Lemnode, G. Grosche, B. Lipphardt, and H. Schnatz, "An optical lattice clock with spin-polarized ${ }^{87}$ Sr atoms", Eur. Phys. J. D 48, pp. 11-17 (2008).

[15] A.D. Ludlow, T. Zelevinsky, G. K. Campbell, S. Blatt, M. M. Boyd, M. H. G. de Miranda, M. J. Martin, J. W. Thomsen, S. M. Foreman, J. Ye, T. M. Fortier, J. E. Stalnaker, S. A. Diddams, Y. Le Coq, Z. W. Barber, N. Poli, N. D. Lemke, K. M. Beck, C. W. Oates, "Sr lattice clock at 10 ${ }^{-16}$ fractional uncertainty by remote optical evaluation with a Ca clock", Science 319, 1805-1808 (2008).

[16] G. K. Campbell, M. M. Boyd, J. W. Thomsen, M. J. Martin, S. Blatt, M. D. Swallows, T. L. Nicholson, T. Fortier, C. W. Oates, S. A. Diddams, "Probing interactions between ultracold fermions", Science 324, $360(2009)$. 